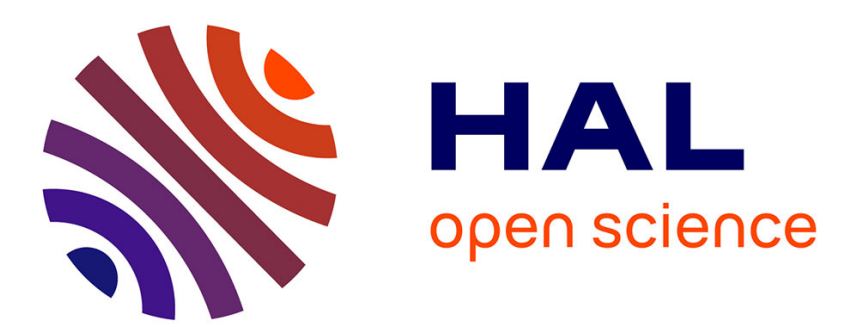

\title{
Migration of an insulating particle under the action of uniform ambient electric and magnetic fields. Part 2. Boundary formulation and ellipsoidal particles
}

Antoine Sellier

\section{- To cite this version:}

Antoine Sellier. Migration of an insulating particle under the action of uniform ambient electric and magnetic fields. Part 2. Boundary formulation and ellipsoidal particles. Journal of Fluid Mechanics, 2003, july (488), pp.335-353. 10.1017/s0022112003004944 . hal-01024937

HAL Id: hal-01024937

https: / hal-polytechnique.archives-ouvertes.fr/hal-01024937

Submitted on 27 Aug 2014

HAL is a multi-disciplinary open access archive for the deposit and dissemination of scientific research documents, whether they are published or not. The documents may come from teaching and research institutions in France or abroad, or from public or private research centers.
L'archive ouverte pluridisciplinaire HAL, est destinée au dépôt et à la diffusion de documents scientifiques de niveau recherche, publiés ou non, émanant des établissements d'enseignement et de recherche français ou étrangers, des laboratoires publics ou privés. 


\title{
Migration of an insulating particle under the action of uniform ambient electric and magnetic fields. Part 2. Boundary formulation and ellipsoidal particles
}

\author{
By A. SELLIER \\ LadHyX, Ecole Polytechnique, 91128 Palaiseau Cédex, France \\ (Received 24 April 2002 and in revised form 28 March 2003)
}

This paper examines the low-Reynolds-number migration of an insulating and rigid particle that is freely suspended in a viscous liquid metal and subject to uniform ambient electric and magnetic fields $\boldsymbol{E}$ and $\boldsymbol{B}$. Under the same physical assumptions as Part 1, a whole boundary formulation of the problem is established. It allows the determination of the particle rigid-body motion without calculating the modified electric field and the flow induced by the Lorentz body force in the fluid domain. The advocated boundary approach, well-adapted for future numerical implementation, makes it possible to obtain an analytical expression for the translational velocity of any ellipsoidal particle (the simplest case of non-spherical orthotropic particles). The behaviour of a spheroid is carefully investigated and discussed both without and with gravity. The migration of this simple non-spherical particle is found to depend on both its nature (prolate or oblate) and the ambient uniform fields $\boldsymbol{E}$ and $\boldsymbol{B}$. The spheroid translates without rotation, and not necessarily parallel to $\boldsymbol{E} \wedge \boldsymbol{B}$. For adequately selected fields $\boldsymbol{E}$ and $\boldsymbol{B}$, the spheroid may either migrate parallel or anti-parallel to a sphere and even be motionless.

\section{Introduction}

As first noticed by Leenov \& Kolin (1954), any solid (conducting or insulating) particle freely suspended in a conducting liquid experiences a rigid-body motion when subject to ambient electric and magnetic fields $\boldsymbol{E}$ and $\boldsymbol{B}$. The rotational Lorentz body force drives a flow which, through viscous effects, induces a migration of the particle. This phenomenon may play a key role in the extraction and/or separation of impurities in liquid metals.

Only spherical and cylindrical particles have been considered by Leenov \& Kolin (1954) and Marty \& Alemany (1984). Since impurities may be non-spherical, it is worth determining the rigid-body motion of small and arbitrarily shaped particles. Within a relevant framework, both the translational velocity $\boldsymbol{U}$ and the angular velocity $\boldsymbol{\Omega}$ of any particle are bilinear in $\boldsymbol{E}$ and $\boldsymbol{B}$ and it has been possible, by exploiting symmetry considerations (Part 1 , Moffatt \& Sellier 2002), to obtain the general form of these bilinear relationships for isotropic, axisymmetric or orthotropic particles. Even for such particles, these relationships involve several unknown geometry-dependent coefficients; a general method is needed to determine these coefficients and also to cope with the case of arbitrary shapes. 


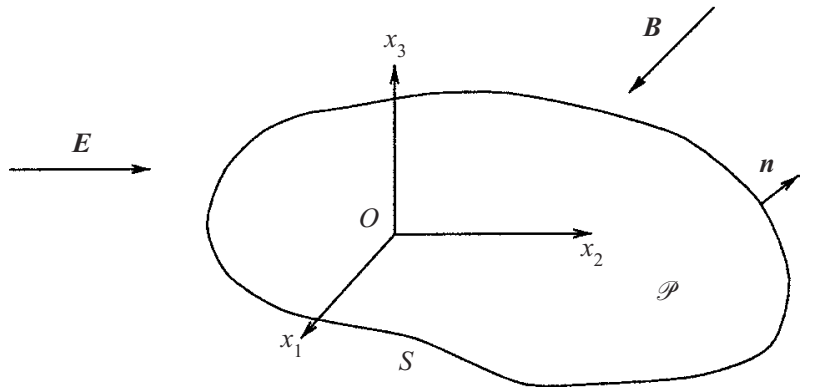

FIGURE 1. An insulating particle embedded in ambient fields $\boldsymbol{E}$ and $\boldsymbol{B}$.

This paper is a continuation of the study initiated in Part 1, under the same physical assumptions of that paper. It presents a general boundary formulation that makes it possible to obtain the rigid-body motion $(\boldsymbol{U}, \boldsymbol{\Omega})$ of any insulating particle. The proposed treatment is analytically worked out for ellipsoidal particles and particular attention is paid to the behaviour of (oblate or prolate) spheroids, both without and with gravity.

The paper is organized as follows. Section $\S 2$ presents our general assumptions and deduces a key governing system for the unknown Cartesian components of the translational and angular velocities. A boundary integral formulation, quite suitable for a future numerical treatment, is established in $\S 3$. The motion of any ellipsoidal particle is obtained analytically in $\S 4$. The detailed behaviour of a spheroid (either oblate or prolate) is addressed and discussed in $\S 5$. Finally, a few concluding remarks in $\S 6$ close the paper.

\section{General assumptions and governing equations}

We consider a solid and insulating particle $\mathscr{P}$ (see figure 1) freely suspended in an unbounded Newtonian liquid metal of uniform density $\rho$, viscosity $\mu$ and conductivity $\sigma>0$. Throughout the paper we adopt Cartesian coordinates $\left(O, x_{1}, x_{2}, x_{3}\right)$, with $O$ the particle centre of volume, and the usual tensor summation convention. For instance, $\boldsymbol{O M}=\boldsymbol{x}=x_{i} \boldsymbol{e}_{i}$ and $r=O M=\left(x_{i} x_{i}\right)^{1 / 2}$.

Far from the particle of length scale $a$, a uniform and steady ambient magnetic field $\boldsymbol{B}$ and a weak enough electric field $\boldsymbol{E}$ are independently and externally imposed. The insulating particle disturbs the ambient field $\boldsymbol{E}$ so that the modified electric field in the whole fluid domain $\Omega$ is $\boldsymbol{E}-\nabla \phi$. The electrostatic perturbation potential $\phi$ is governed by the well-posed exterior Neumann problem

$$
\nabla^{2} \phi=0 \text { in } \Omega, \quad \nabla \phi \rightarrow \mathbf{0} \quad \text { as } r \rightarrow \infty, \quad \nabla \phi \cdot \boldsymbol{n}=\boldsymbol{E} \cdot \boldsymbol{n} \text { on } S,
$$

where $\boldsymbol{n}$ denotes the unit outward normal on the smooth enough particle's surface $S$. As explained in Part 1, the magnetic field $\boldsymbol{B}$ is not affected by the presence of the particle and the resulting Lorentz body force $\boldsymbol{f}=\boldsymbol{j} \wedge \boldsymbol{B}$, where $\boldsymbol{j}$ is the current, drives a steady Stokes flow $(\boldsymbol{u}, p)$ of scale $U$. The particle has a rigid-body motion, with velocity $\boldsymbol{U}$ of its centre of volume $O$ and angular velocity $\boldsymbol{\Omega}$. The Reynolds number $R e=\rho U a / \mu$ is assumed to be small and under the assumptions set in Part 1, the flow $(\boldsymbol{u}, p)$ obeys the governing problem

$$
\nabla \cdot \boldsymbol{u}=0, \mu \nabla^{2} \boldsymbol{u}=\nabla p-\boldsymbol{f} \text { in } \Omega, \quad(\boldsymbol{u}, p) \rightarrow\left(\mathbf{0}, p_{\infty}\right) \quad \text { as } r \rightarrow \infty, \quad \boldsymbol{u}=\boldsymbol{u}_{d} \text { on } S
$$


with the following definitions

$$
\boldsymbol{f}=\sigma(\boldsymbol{E}-\nabla \phi) \wedge \boldsymbol{B}, \quad p_{\infty}=(\sigma \boldsymbol{E} \wedge \boldsymbol{B}) \cdot \boldsymbol{x}, \quad \boldsymbol{u}_{d}=\boldsymbol{U}+\boldsymbol{\Omega} \wedge \boldsymbol{x} .
$$

The linear pressure $p_{\infty}$ relates to the case of a quiescent fluid with uniform body force $\boldsymbol{f}_{\infty}=\sigma \boldsymbol{E} \wedge \boldsymbol{B}$. The combination of problems (2.1) and (2.2)-(2.3) makes it possible to express the fluid stress tensor $\sigma=\sigma(\boldsymbol{u}, p)$ in terms of the unknown velocities $\boldsymbol{U}$ and $\boldsymbol{\Omega}$. For a freely suspended particle, these unknown vectors are finally obtained by requiring zero net hydrodynamic force $\boldsymbol{F}_{\text {net }}$ and torque $\boldsymbol{G}_{\text {net }}$ on $S$. Thus, equations (2.1), (2.2)-(2.3) are supplemented with the conditions

$$
\boldsymbol{F}_{\text {net }}=\int_{S} \boldsymbol{\sigma} \cdot \boldsymbol{n} \mathrm{d} S=\mathbf{0}, \quad \boldsymbol{G}_{\text {net }}=\int_{S} \boldsymbol{x} \wedge[\boldsymbol{\sigma} \cdot \boldsymbol{n}] \mathrm{d} S=\mathbf{0} .
$$

By linearity it is both possible and fruitful to decompose the fluid motion $(\boldsymbol{u}, p)$ into three Stokes flows $\left(\boldsymbol{u}^{(1)}, p^{(1)}\right),\left(\boldsymbol{u}^{(2)}, p^{(2)}\right)$ and $\left(\boldsymbol{u}^{(3)}, p^{(3)}\right)$ that fulfil (2.2) for the following settings:

$$
\begin{gathered}
\boldsymbol{f}^{(1)}=\boldsymbol{f}_{\infty}=\sigma \boldsymbol{E} \wedge \boldsymbol{B}, \quad p_{\infty}^{(1)}=\boldsymbol{f}_{\infty} \cdot \boldsymbol{x}, \quad \boldsymbol{u}_{d}^{(1)}=\mathbf{0}, \\
\boldsymbol{f}^{(2)}=-\sigma \nabla \phi \wedge \boldsymbol{B}, \quad p_{\infty}^{(2)}=0, \quad \boldsymbol{u}_{d}^{(2)}=\mathbf{0}, \\
\boldsymbol{f}^{(3)}=\mathbf{0}, \quad p_{\infty}^{(3)}=0, \quad \boldsymbol{u}_{d}^{(3)}=\boldsymbol{U}+\boldsymbol{\Omega} \wedge \boldsymbol{x} .
\end{gathered}
$$

Let us introduce, for $n \in\{1,2,3\}$, the resulting force $\boldsymbol{F}^{(n)}=\int_{S} \boldsymbol{\sigma}^{(n)} \cdot \boldsymbol{n} \mathrm{d} S$ and torque $\boldsymbol{G}^{(n)}=\int_{S} \boldsymbol{x} \wedge\left[\boldsymbol{\sigma}^{(n)} \cdot \boldsymbol{n}\right] \mathrm{d} S$ on the particle. The electromagnetic net force $\boldsymbol{F}^{(1)}$ and torque $\boldsymbol{G}^{(1)}$ are exerted by the uniform ambient Lorentz body force $\sigma \boldsymbol{E} \wedge \boldsymbol{B}$ whilst additional electromagnetic net force $\boldsymbol{F}^{(2)}$ and torque $\boldsymbol{G}^{(2)}$ are produced by the induced Lorentz body force $-\sigma \nabla \phi \wedge \boldsymbol{B}$. As previously noted, $\boldsymbol{u}^{(1)}=\mathbf{0}$ and $p^{(1)}=\boldsymbol{f}_{\infty} \cdot \boldsymbol{x}$. Thus, $\boldsymbol{\sigma}^{(1)} \cdot \boldsymbol{n}=$ $-\left[\boldsymbol{f}_{\infty} \cdot \boldsymbol{x}\right] \boldsymbol{n}$ and, using elementary algebra, we arrive at

$$
\boldsymbol{F}^{(1)}=-\sigma \mathscr{V}_{\mathscr{P}}[\boldsymbol{E} \wedge \boldsymbol{B}], \quad \boldsymbol{G}^{(1)}=\sigma[\boldsymbol{E} \wedge \boldsymbol{B}] \wedge \int_{\mathscr{P}} \boldsymbol{x} \mathrm{d} v,
$$

where $\mathscr{V}_{\mathscr{P}}$ denotes the volume of the particle. In dealing with the remaining forces $\boldsymbol{F}^{(n)}$ and torques $\boldsymbol{G}^{(n)}$, it is worth extending the usual Lorentz reciprocal theorem (Kim \& Karrila 1991) as follows.

THEOREM 1. If two fluid motions $(\boldsymbol{u}, p)$ and $\left(\boldsymbol{u}^{\prime}, p^{\prime}\right)$ (of associated stress tensors $\boldsymbol{\sigma}$ and $\boldsymbol{\sigma}^{\prime}$ ) obey the equations (2.2) with, for two real values $a>2$ and $a^{\prime}>2$, the basic behaviours

$$
r^{a}|\boldsymbol{f}| \rightarrow 0,(\boldsymbol{u}, p) \rightarrow(\mathbf{0}, 0), r^{a^{\prime}}\left|\boldsymbol{f}^{\prime}\right| \rightarrow 0 \quad \text { and } \quad\left(\boldsymbol{u}^{\prime}, p^{\prime}\right) \rightarrow(\mathbf{0}, 0) \text { as } r \rightarrow \infty
$$

then the following key relation holds

$$
\int_{S}\left[\boldsymbol{u}_{d} \cdot \boldsymbol{\sigma}^{\prime} \cdot \boldsymbol{n}-\boldsymbol{u}_{d}^{\prime} \cdot \boldsymbol{\sigma} \cdot \boldsymbol{n}\right] \mathrm{d} S=-\int_{\Omega}\left[\boldsymbol{u}^{\prime} \cdot \boldsymbol{f}-\boldsymbol{u} \cdot \boldsymbol{f}^{\prime}\right] \mathrm{d} \Omega .
$$

Resorting to the far-field expansion of equations (2.2), as in Lamb (1932), we indeed find that, far from the particle, the fluid velocity decays at least as fast as $1 / r$ whilst both the pressure $p$ and the stress tensor $\sigma$ decay at least as fast as $1 / r^{2}$. The establishment of (2.10) makes use of such 'good' behaviours of the body forces and fluid flows at infinity and actually consists in a straightforward extension of the available proofs for zero body forces $f$ and $\boldsymbol{f}^{\prime}$ (among others, see Kim \& Karrila 1991; Pozrikidis 1992). Actually, (2.10) is available in Teubner (1982), but we here specify suitable far-field behaviours (2.9) for the body forces $\boldsymbol{f}$ and $\boldsymbol{f}^{\prime}$. In exploiting 
the identity (2.10), we identify $(\boldsymbol{u}, p)$ with one of the usual (Happel \& Brenner 1973) translational $\left(\boldsymbol{u}_{T}^{(i)}, p_{T}^{(i)}\right)$ or rotational $\left(\boldsymbol{u}_{R}^{(i)}, p_{R}^{(i)}\right)$ motions that obey equations $(2.2)$ for a zero body force, $p_{\infty}=0$ and the boundary conditions

$$
\boldsymbol{u}_{T}^{(i)}=\boldsymbol{e}_{i}, \quad \boldsymbol{u}_{R}^{(i)}=\boldsymbol{e}_{i} \wedge \boldsymbol{x} \quad \text { on } S .
$$

Under the admissible choice $\left(\boldsymbol{u}^{\prime}, p^{\prime}\right)=\left(\boldsymbol{u}^{(3)}, p^{(3)}\right)$, our result (2.10) (which reduces here to the standard reciprocal theorem) immediately yields the widely employed relations

$$
\boldsymbol{F}^{(3)}=-\mu\{\boldsymbol{K} \cdot \boldsymbol{U}+\boldsymbol{V} \cdot \boldsymbol{\Omega}\}, \quad \boldsymbol{G}^{(3)}=-\mu\{\boldsymbol{D} \cdot \boldsymbol{U}+\boldsymbol{W} \cdot \boldsymbol{\Omega}\}
$$

where the Cartesian components of the usual translation tensor $\boldsymbol{K}$, rotation tensor $\boldsymbol{W}$ and coupling tensors $\boldsymbol{V}$ and $\boldsymbol{D}$ satisfy

$$
\begin{aligned}
-\mu K_{i j} & =\int_{S} \boldsymbol{e}_{j} \cdot \boldsymbol{f}_{T}^{(i)} \mathrm{d} S, \quad-\mu W_{i j}=\int_{S}\left[\boldsymbol{e}_{j} \wedge \boldsymbol{x}\right] \cdot \boldsymbol{f}_{R}^{(i)} \mathrm{d} S, \\
-\mu V_{i j} & =\int_{S}\left[\boldsymbol{e}_{j} \wedge \boldsymbol{x}\right] \cdot \boldsymbol{f}_{T}^{(i)} \mathrm{d} S, \quad-\mu D_{i j}=\int_{S} \boldsymbol{e}_{j} \cdot \boldsymbol{f}_{R}^{(i)} \mathrm{d} S,
\end{aligned}
$$

if, for $L \in\{T, R\}$ and $i \in\{1,2,3\}$, the vector $\boldsymbol{f}_{L}^{(i)}=\boldsymbol{\sigma}\left(\boldsymbol{u}_{L}^{(i)}, p_{L}^{(i)}\right) \cdot \boldsymbol{n}$ stands for the surface force that is exerted on the particle $\mathscr{P}$ for the fluid motion $\left(\boldsymbol{u}_{L}^{(i)}, p_{L}^{(i)}\right)$.

Now we need to calculate $\boldsymbol{F}^{(2)}$ and $\boldsymbol{G}^{(2)}$. Since $\nabla \phi \cdot \boldsymbol{n}=\boldsymbol{E} \cdot \boldsymbol{n}$ on $S$ with $\int_{S} \boldsymbol{E} \cdot \boldsymbol{n} \mathrm{d} S=0$, note that $\phi \sim 1 / r^{q}$ as $r \rightarrow \infty$ with $q \geqslant 2$. Accordingly, the body force $\boldsymbol{f}^{(2)}$ satisfies the far-field property $\left|r^{a^{\prime}} \boldsymbol{f}^{(2)}\right| \rightarrow 0$ with $a^{\prime}>2$. The legitimate application of theorem 1 to the flow $\left(\boldsymbol{u}^{\prime}, p^{\prime}\right)=\left(\boldsymbol{u}^{(2)}, p^{(2)}\right)$ provides the basic relations

$$
\begin{aligned}
& F_{i}^{(2)}=\boldsymbol{F}^{(2)} \cdot \boldsymbol{e}_{i}=-\sigma \int_{\Omega} \boldsymbol{u}_{T}^{(i)} \cdot[\nabla \phi \wedge \boldsymbol{B}] \mathrm{d} \Omega, \\
& G_{i}^{(2)}=\boldsymbol{G}^{(2)} \cdot \boldsymbol{e}_{i}=-\sigma \int_{\Omega} \boldsymbol{u}_{R}^{(i)} \cdot[\nabla \phi \wedge \boldsymbol{B}] \mathrm{d} \Omega .
\end{aligned}
$$

By virtue of (2.8) and (2.12), the conditions (2.4) yield the key system

$$
\begin{gathered}
\boldsymbol{K} \cdot \boldsymbol{U}+\boldsymbol{V} \cdot \boldsymbol{\Omega}=\frac{1}{\mu}\left\{-\sigma \mathscr{V}_{\mathscr{P}}[\boldsymbol{E} \wedge \boldsymbol{B}]+F_{i}^{(2)} \boldsymbol{e}_{i}\right\}=\frac{1}{\mu} \boldsymbol{F}^{E}, \\
\boldsymbol{D} \cdot \boldsymbol{U}+\boldsymbol{W} \cdot \boldsymbol{\Omega}=\frac{1}{\mu}\left\{\sigma[\boldsymbol{E} \wedge \boldsymbol{B}] \wedge \int_{\mathscr{P}} \boldsymbol{x} \mathrm{d} v+G_{i}^{(2)} \boldsymbol{e}_{i}\right\}=\frac{1}{\mu} \boldsymbol{G}^{E},
\end{gathered}
$$

where the electromagnetic force $\boldsymbol{F}^{E}=\boldsymbol{F}^{(1)}+\boldsymbol{F}^{(2)}$ and torque $\boldsymbol{G}^{E}=\boldsymbol{G}^{(1)}+\boldsymbol{G}^{(2)}$, introduced as in Part 1, are obtained from (2.8) and (2.15)-(2.16). Note that both tensors $\boldsymbol{K}$ and $\boldsymbol{W}$ are symmetric whereas $\boldsymbol{V}$ is the transpose of $\boldsymbol{D}$ (invoke, once more, the identity (2.10)). Thus, the resistance matrix, $\boldsymbol{M}$, associated with the linear system (2.17)-(2.18) and defined, as in Happel \& Brenner (1973), by

$$
\boldsymbol{M}=\left(\begin{array}{ll}
\boldsymbol{K} & \boldsymbol{V} \\
\boldsymbol{D} & \boldsymbol{W}
\end{array}\right)
$$

is symmetric. In addition, the requirement of positive energy dissipation (Happel \& Brenner 1973) guarantees that $\boldsymbol{M}$ is also positive-definite. Hence, the governing system (2.17)-(2.18) admits a unique solution $(\boldsymbol{U}, \boldsymbol{\Omega})$ whose determination consists of the following steps:

(i) Evaluate the previous tensors $\boldsymbol{K}, \boldsymbol{W}, \boldsymbol{V}$ and $\boldsymbol{D}$. Such a standard task is either achieved by directly (see (3.1)) determining the surface forces $f_{L}^{(i)}$ on $S$ or after computing the specific flows $\left(\boldsymbol{u}_{L}^{(i)}, p_{L}^{(i)}\right)$ in the whole fluid domain. 
(ii) Determine the force and torque components $F_{i}^{(2)}$ and $G_{i}^{(2)}$. This is our key problem. At first glance, the relations (2.15) suggest that it may be necessary to compute the electric perturbation field $-\nabla \phi$ and the velocities $\boldsymbol{u}_{T}^{(i)}$ and $\boldsymbol{u}_{R}^{(i)}$ in the whole domain $\Omega$. Fortunately, as shown in $\S 3$, we actually only need to evaluate $\phi$ and its derivatives (up to order two) on the surface $S$.

\section{A boundary formulation}

In this key section we prove that, in determining $(\boldsymbol{U}, \boldsymbol{\Omega})$, it is enough to know the surface forces $\boldsymbol{f}_{T}^{(i)}, \boldsymbol{f}_{R}^{(i)}$ and the derivatives up to order two of the perturbation potential $\phi$ on the particle's surface $S$.

The procedure that we advocate rests on the key boundary representation, in terms of a single-layer potential, of any velocity field $\boldsymbol{u}_{L}^{(i)}$ in the whole fluid domain $\Omega$. More precisely, for given $i \in\{1,2,3\}$ and $L \in\{T, R\}$, the following representation holds (Pozrikidis 1992)

$$
\left[\boldsymbol{u}_{L}^{(i)} \cdot \boldsymbol{e}_{j}\right](M)=-\frac{1}{8 \pi \mu} \int_{S}\left[\boldsymbol{f}_{L}^{(i)} \cdot \boldsymbol{e}_{k}\right](P) G_{j k}(P, M) \mathrm{d} S_{P},
$$

for $M \in \Omega \cup S$, where

$$
G_{j k}(P, M)=\frac{\delta_{j k}}{P M}+\frac{\left(\boldsymbol{P M} \cdot \boldsymbol{e}_{j}\right)\left(\boldsymbol{P M} \cdot \boldsymbol{e}_{k}\right)}{P M^{3}} .
$$

Applying (3.1) for $M$ on $S$ and using the boundary conditions (2.11), we arrive at a Fredholm boundary integral equation of the first kind for the surface force $f_{L}^{(i)}$. This integral equation admits a solution unique up to any constant multiple of the outward normal $\boldsymbol{n}$ on $S$ (Ladyzhenskaya 1969). From (2.11) and (3.1) we may then find the surface forces $\boldsymbol{f}_{L}^{(i)}$ and the resistance matrix $\boldsymbol{M}$ defined by (2.19).

For $r$ large enough, let us denote by $\Omega(r)$ the fluid volume that is enclosed between the particle surface $S$ and the surface $S_{r}:=\{M, O M=r\}$ and introduce the integrals $I_{L}^{(i)}$ and $I_{L}^{(i)}(r)$ as

$$
I_{L}^{(i)}=\lim _{r \rightarrow \infty}\left[I_{L}^{(i)}(r)\right], \quad I_{L}^{(i)}(r)=-8 \pi \mu \int_{\Omega(r)} \boldsymbol{u}_{L}^{(i)}(M) \cdot[\nabla \phi \wedge \boldsymbol{B}](M) \mathrm{d} \Omega .
$$

As detailed in Appendix A, we thus arrive at the key decomposition

$$
I_{L}^{(i)}=\mathscr{L}\left[\boldsymbol{f}_{L}^{(i)}\right], \quad \mathscr{L}=\mathscr{L}_{1}+\mathscr{L}_{2}+\mathscr{L}_{3},
$$

where the linear operators $\mathscr{L}_{k}$ are defined, for any field $\boldsymbol{v}$ on $S$, as follows

$$
\begin{gathered}
\mathscr{L}_{1}[\boldsymbol{v}]=-\int_{S} \int_{S} \boldsymbol{v}(P) \cdot[\nabla \phi(M) \wedge \boldsymbol{B}] \frac{\boldsymbol{P M} \cdot \boldsymbol{n}(M)}{P M} \mathrm{~d} S_{P} \mathrm{~d} S_{M}, \\
\mathscr{L}_{2}[\boldsymbol{v}]=\int_{S} \int_{S}\left[\boldsymbol{v}(P) \cdot \frac{\boldsymbol{P M}}{P M}\right][\nabla \phi(M) \wedge \boldsymbol{B}] \cdot \boldsymbol{n}(M) \mathrm{d} S_{P} \mathrm{~d} S_{M}, \\
\mathscr{L}_{3}[\boldsymbol{v}]=\int_{S} \int_{S} \epsilon_{k m n} P M\left[\boldsymbol{v} \cdot \boldsymbol{e}_{k}\right](P)\left[\boldsymbol{B} \cdot \boldsymbol{e}_{n}\right]\left[\phi_{, m l}\left(\boldsymbol{n} \cdot \boldsymbol{e}_{l}\right)\right](M) \mathrm{d} S_{P} \mathrm{~d} S_{M},
\end{gathered}
$$

if the notation $\phi_{m l}=\partial^{2} \phi / \partial x_{m} \partial x_{l}$ is adopted and $\epsilon_{k m n}$ denotes the permutation tensor. Both operators $\mathscr{L}_{1}$ and $\mathscr{L}_{2}$ involve the perturbation field $\nabla \phi$ on $S$ whereas $\mathscr{L}_{3}$ requires the evaluation of second-order derivatives of $\phi$ on the surface $S$. By virtue of (3.4), the basic relations (2.15)-(2.16) become

$$
F_{i}^{(2)}=\sigma \mathscr{L}\left[\boldsymbol{f}_{T}^{(i)}\right] /(8 \pi \mu), \quad G_{i}^{(2)}=\sigma \mathscr{L}\left[\boldsymbol{f}_{R}^{(i)}\right] /(8 \pi \mu) .
$$


As previously stated, the determination of the surface forces $f_{L}^{(i)}$ and the derivatives, up to order two, of the perturbation potential $\phi$ on the particle's surface allows us to calculate the resistance matrix $\boldsymbol{M}$ (use (2.13)-(2.14)) and the vectors $\boldsymbol{F}^{(2)}$ and $\boldsymbol{G}^{(2)}$ (invoke (3.4)-(3.8)). Unfortunately, the analytical evaluation of such quantities seems feasible only for a very few shapes (for instance, the tractable case of the ellipsoidal particle is addressed in detail in $\S 4$ ). For a particle of arbitrary (but smooth enough) boundary, a numerical treatment is required. However, the important message is that we only need to approximate the solutions of the Fredholm boundary integral equations on $S$. In other words, for a numerical implementation, it is unnecessary to compute the fields $\boldsymbol{u}_{L}^{(i)}$ and $\nabla \phi$ in the whole fluid domain $\Omega$ (as suggested at the end of $\S 2$ ). More precisely, a possible numerical strategy consists of the following steps:

(i) Approximate the solutions $f_{L}^{(i)}$ to integral equations (3.1) (with $M$ on $S$ and for prescribed conditions (2.11)) by using a standard boundary-element method (see, for instance, Brebbia, Telles \& Wrobel 1984; Beskos 1987; Bonnet 1999). This makes it possible to compute the resistance matrix $\boldsymbol{M}$.

(ii) Evaluate the function $\phi$ on $S$ by solving the well-known Fredholm boundaryintegral equation of the second kind

$$
-4 \pi \phi(M)+\int_{S}[\phi(P)-\phi(M)] \frac{\boldsymbol{P M} \cdot \boldsymbol{n}(P)}{P M^{3}} \mathrm{~d} S=\int_{S} \frac{[\boldsymbol{E} \cdot \boldsymbol{n}](P)}{P M} \mathrm{~d} S .
$$

This well-posed equation easily follows from the governing problem (2.1). By resorting to high enough-order boundary elements on $S$ (at least when dealing with (3.9)), we may then build numerical approximations of the required derivatives $\phi_{, i}$ and $\phi_{, i j}$ on $S$.

\section{The migration of ellipsoidal particles}

Implementing the proposed numerical procedure makes it possible to approximate the required rigid-body motion $(\boldsymbol{U}, \boldsymbol{\Omega})$ for any particle, a task postponed to a future paper (Part 3). Both for theoretical reasons and numerical benchmarks it, however, remains of prime interest to obtain, whenever possible, analytical solutions. The spherical particle provides a natural candidate for such theoretical investigations. The case of a fixed and insulating or conducting sphere has been previously addressed by Leenov \& Kolin (1954) in their pioneering work and further handled by Marty $\&$ Alemany (1984). Those works actually calculate the Stokes fluid motion and the perturbation potential $\phi$ in the whole fluid domain. As reported in this section, our approach makes it possible to analytically obtain the motion $(\boldsymbol{U}, \boldsymbol{\Omega})$ in the more tricky case of ellipsoidal particles. Before detailing our results, we briefly present the expected behaviour of such orthotropic particles (as predicted in Part 1) and introduce the 'mobility' pseudo-tensor $\boldsymbol{S}$.

\subsection{General behaviour of orthotropic particles}

This subsection focuses on the case of an orthotropic particle and makes use of the symmetry properties of the electromagnetic force $\boldsymbol{F}^{E}$ and torque $\boldsymbol{G}^{E}$. Since it is orthotropic, the particle exhibits three mutually orthogonal planes of symmetry that intersect at its centre of volume $O$. Selecting vectors $\boldsymbol{e}_{i}$ normal to these planes of symmetry, we immediately obtain (see Happel \& Brenner 1973) diagonal tensors $\boldsymbol{K}$ and $\boldsymbol{W}$ and zero coupling tensors $\boldsymbol{V}$ and $\boldsymbol{D}$. Under our notation, it follows that (with a summation over suffixes $i \in\{1,2,3\})$

$$
\boldsymbol{K}=K_{i i} \boldsymbol{e}_{i} \otimes \boldsymbol{e}_{i}, \quad \boldsymbol{W}=W_{i i} \boldsymbol{e}_{i} \otimes \boldsymbol{e}_{i}, \quad \boldsymbol{E}=E_{i} \boldsymbol{e}_{i}, \quad \boldsymbol{B}=B_{i} \boldsymbol{e}_{i} .
$$


Owing to the requirement of positive mechanical energy dissipation, note that $K_{i i}>0$ and $W_{i i}>0$. For any orthotropic particle (Part 1) symmetry considerations actually show that $\boldsymbol{G}^{E}$ is zero whereas $\boldsymbol{F}^{E}=F_{i}^{E} \boldsymbol{e}_{i}$ reads

$$
F_{i}^{E}=\sigma a^{3} A_{i j k} E_{j} B_{k} \quad \text { with } \quad A_{i j k}=0 \text { if } \epsilon_{i j k}=0 .
$$

Accordingly, there exists a third-rank volume pseudo-tensor $\boldsymbol{A}^{\prime}$ whose Cartesian components $A_{i j k}^{\prime}$ vanish with $\epsilon_{i j k}$ and obey

$$
F_{i}^{(2)}=\sigma A_{i j k}^{\prime} E_{j} B_{k}, \quad A_{i j k}^{\prime}=a^{3} A_{i j k}+\epsilon_{i j k} \mathscr{V} \mathscr{P} .
$$

Recall that $\boldsymbol{F}^{(2)}$ is produced by the induced Lorentz body force $-\sigma \nabla \phi \wedge \boldsymbol{B}$. In view of (4.1), governing equations (2.17)-(2.18) decouple and the orthotropic particle translates (without rotation) at the velocity $\boldsymbol{U}$ given by

$$
U_{i}=\boldsymbol{U} \cdot \boldsymbol{e}_{i}=\frac{\sigma\left(A_{i j k}^{\prime}-\mathscr{V}_{\mathscr{P}} \epsilon_{i j k}\right)\left[\boldsymbol{E} \cdot \boldsymbol{e}_{j}\right]\left[\boldsymbol{B} \cdot \boldsymbol{e}_{k}\right]}{\mu K_{i i}} .
$$

In other words, there exists a surface third-rank 'mobility' pseudo-tensor $\boldsymbol{S}$ of Cartesian components $S_{i j k}$ such that

$$
U_{i}=\frac{\sigma}{\mu} S_{i j k}\left[\boldsymbol{E} \cdot \boldsymbol{e}_{j}\right]\left[\boldsymbol{B} \cdot \boldsymbol{e}_{k}\right], \quad S_{i j k}=\frac{A_{i j k}^{\prime}-\mathscr{V}_{\mathscr{P}} \epsilon_{i j k}}{K_{i i}} .
$$

Each quantity $S_{i j k}$ has the dimension of a surface and the key pseudo-tensor $\boldsymbol{S}$ is determined solely by the geometry of the orthotropic particle. As obtained in Part 1, if $\boldsymbol{E}$ and $\boldsymbol{B}$ are normal to the same plane of symmetry, the orthotropic particle does not translate and it moves parallel to $\boldsymbol{E} \wedge \boldsymbol{B}$ as soon as $\boldsymbol{E}$ and $\boldsymbol{B}$ are normal to two different planes of symmetry. For a non-isotropic and orthotropic particle at least one sum $S_{i j k}+S_{i k j}$ is non-zero and thereby $\boldsymbol{U}$ is not necessarily parallel to $\boldsymbol{E} \wedge \boldsymbol{B}$ when $\boldsymbol{E}$ or $\boldsymbol{B}$ is not normal to a plane of symmetry. However, observe that if $S_{i j k}+S_{i k j}=0$ for $\epsilon_{i j k}={ }_{-}^{+} 1$ then $\boldsymbol{U}=\sigma S_{i j k}(\boldsymbol{E} \wedge \boldsymbol{B}) / \mu$ as soon as $\boldsymbol{E} \cdot \boldsymbol{e}_{i}=\boldsymbol{B} \cdot \boldsymbol{e}_{i}=0$.

\subsection{The 'mobility' pseudo-tensor of an ellipsoid}

We address the case of the ellipsoidal particle and calculate the associated 'mobility' pseudo-tensor $\boldsymbol{S}$. Henceforth, $\mathscr{E}_{\lambda}$ denotes, for $\lambda \geqslant 0$, the ellipsoid of equation

$$
x_{1}^{2} /\left(a_{1}^{2}+\lambda\right)+x_{2}^{2} /\left(a_{2}^{2}+\lambda\right)+x_{3}^{2} /\left(a_{3}^{2}+\lambda\right)=1
$$

if $\boldsymbol{O M}=x_{i} \boldsymbol{e}_{i}$ and we consider the ellipsoidal particle $\mathscr{P}$ of boundary $\mathscr{E} 0$, i.e. such that

$$
x_{1}^{2} / a_{1}^{2}+x_{2}^{2} / a_{2}^{2}+x_{3}^{2} / a_{3}^{2} \leqslant 1 \text { for } M\left(x_{1}, x_{2}, x_{3}\right) \in \mathscr{P} .
$$

For symmetry reasons, we confine the analysis to the ambient fields $\boldsymbol{B}=B_{1} \boldsymbol{e}_{1}, \boldsymbol{E}=$ $E_{2} \boldsymbol{e}_{2}$ and look at the only non-zero velocity component $U_{3}=\boldsymbol{U} \cdot \boldsymbol{e}_{3}$. According to (3.8), it is sufficient to evaluate the surface force $\boldsymbol{f}_{T}^{(3)}$. As established in Jeffery (1922), this vector takes (up to a constant multiple of the outward normal $\boldsymbol{n}$ ) the simple form

$$
\boldsymbol{f}_{T}^{(3)}(M)=A^{(3)} s(M) \boldsymbol{e}_{3}, \quad A^{(3)}=-4 \mu /\left[\chi+a_{3}^{2} \alpha_{3}\right]
$$

with, for $i \in\{1,2,3\}$, the following definitions

$$
\begin{gathered}
s(M)=\left\{x_{1}^{2} / a_{1}^{4}+x_{2}^{2} / a_{2}^{4}+x_{3}^{2} / a_{3}^{4}\right\}^{-1 / 2}, \quad \Xi(t)=\left(a_{1}^{2}+t\right)\left(a_{2}^{2}+t\right)\left(a_{3}^{2}+t\right), \\
\chi=a_{1} a_{2} a_{3} \int_{0}^{\infty} \frac{\mathrm{d} t}{\Delta(t)}, \quad \alpha_{i}=a_{1} a_{2} a_{3} \int_{0}^{\infty} \frac{\mathrm{d} t}{\left(a_{i}^{2}+t\right) \Delta(t)}, \quad \Delta(t)=\sqrt{\Xi(t)} .
\end{gathered}
$$


For $i \in\{1,2,3\}$ and at any point $M\left(x_{1}, x_{2}, x_{3}\right)$ it is both legitimate and fruitful to introduce the integrals

$$
I_{i}(M)=\int_{\mathscr{\delta}_{0}} \frac{\left(x_{i}-x_{i}^{P}\right) s(P)}{P M} \mathrm{~d} S_{P}, \quad J(M)=\int_{\mathscr{E}_{0}} P M s(P) \mathrm{d} S_{P} .
$$

Indeed, if we set $T_{k}=\mathscr{L}_{k}\left[\boldsymbol{f}_{T}^{(3)}\right]$, the combination of definitions (3.5)-(3.7) and above results (4.8)-(4.10) or definitions (4.11) shows that

$$
T_{2}=B_{1} A^{(3)} \int_{\mathscr{E}_{0}} \epsilon_{l m 1}\left[\phi,{ }_{m} I_{3} \boldsymbol{n} \cdot \boldsymbol{e}_{l}\right] \mathrm{d} S, \quad T_{1}+T_{3}=B_{1} A^{(3)} \int_{\mathscr{E}_{0}}\left[\phi,{ }_{2} I_{l}-\phi,,_{2 l} J\right]\left[\boldsymbol{n} \cdot \boldsymbol{e}_{l}\right] \mathrm{d} S .
$$

Thus, the calculation of the force component $F_{3}^{(2)}=\sigma\left[T_{1}+T_{2}+T_{2}\right] /(8 \pi \mu)$ reduces to the determination of functions $J, I_{i}, \phi_{, l}$ and $\phi_{, 2 l}$ on the particle's surface $\mathscr{E}_{0}$. This task is achieved in the following steps.

Step 1. Evaluation of integrals $I_{i}$ inside the ellipsoid and on its boundary.

As proved in the Appendix, we obtain, both inside the ellipsoid and on its surface,

$$
I_{i}(M)=I_{i}\left(x_{1}, x_{2}, x_{3}\right)=Q_{i} x_{i}, \quad Q_{i}=2 \pi\left[\chi-\alpha_{i} a_{i}^{2}\right]
$$

where there is no summation over suffixes $k$.

Step 2. Calculation of $\phi$ and $\phi_{, m}$ on $\mathscr{E}_{0}$ and evaluation of $T_{2}$.

For our ellipsoidal particle the perturbation potential $\phi$, solution to problem (2.1) with $\boldsymbol{E}=E_{2} \boldsymbol{e}_{2}$, admits in the whole flow domain $\Omega$ the well-known form (Lamb 1932)

$$
\phi(M)=\phi\left(\lambda_{e}\right)=\left[\frac{a_{1} a_{2} a_{3} E_{2}}{\alpha_{2}-2}\right] \phi^{\prime}\left(\lambda_{e}\right), \quad \phi^{\prime}\left(\lambda_{e}\right)=x_{2} \int_{\lambda_{e}}^{\infty} \frac{\mathrm{d} t}{\left(a_{2}^{2}+t\right) \Delta(t)} .
$$

Each required derivative $\phi^{\prime},{ }_{m}$ is obtained on $\mathscr{E}_{0}$ as explained in the Appendix. Our definition (4.12) of $T_{2}$ thereby yields

$$
T_{2}=-B_{1} E_{2} Q_{3} A^{(3)}\left[\frac{a_{1} a_{2} a_{3}}{\alpha_{2}-2}\right]\left[\int_{0}^{\infty} \frac{\mathrm{d} t}{\left(a_{2}^{2}+t\right) \Delta(t)}\right]\left[\int_{\mathscr{E}_{0}} \frac{x_{3}^{2} s(M)}{a_{3}^{2}} \mathrm{~d} S_{M}\right] .
$$

Noting that $s(M) \mathrm{d} S_{M}=a_{1} a_{2} a_{3} \sin \varphi \mathrm{d} \varphi \mathrm{d} \theta$ for ellipsoidal coordinates $\varphi \in[0, \pi]$ and $\theta \in[0,2 \pi]$ such that $x_{3}=a_{3} \cos \varphi, x_{2}=x_{2} \sin \varphi \sin \theta$ and $x_{1}=a_{1} \sin \varphi \cos \theta$, we finally arrive at

$$
T_{2}=-\frac{4}{3} \pi \frac{a_{1} a_{2} a_{3} \alpha_{2}}{\left[\alpha_{2}-2\right]} B_{1} E_{2} Q_{3} A^{(3)} .
$$

Step 3. Calculation of $T_{1}+T_{3}$.

For the sake of conciseness, details are displayed in Appendix B. We obtain

$$
T_{1}+T_{3}=\frac{16 \pi^{2} a_{1} a_{2} a_{3}}{3\left[\alpha_{2}-2\right]} B_{1} E_{2} A^{(3)}\left[\chi \alpha_{2}+\alpha_{2} a_{2}^{2}-\chi\right] .
$$

Combining results (4.13) and (4.17) makes it possible to compute $F_{3}^{(2)}$. Finally, definitions (2.13) and (4.3) in conjunction with (4.8) yield

$$
\frac{A_{321}^{\prime}}{K_{33}}=-\frac{\epsilon_{321}}{12\left[\alpha_{2}-2\right]}\left\{2\left(\chi-\alpha_{2} a_{2}^{2}\right)-\alpha_{2}\left(\chi+\alpha_{3} a_{3}^{2}\right)\right\} .
$$

Therefore, as the reader may easily check by using cyclic interchanges of suffixes, the Cartesian component $S_{i j k}$ of the 'mobility' pseudo-tensor $\boldsymbol{S}$ becomes (no summation 
over suffices $i$ and $j$ in (4.19))

$$
S_{i j k}=\frac{\epsilon_{i j k}}{12}\left\{\frac{\alpha_{j}\left(2 a_{j}^{2}+\alpha_{i} a_{i}^{2}\right)}{\left[\alpha_{j}-2\right]}-\alpha_{i} a_{i}^{2}\right\} .
$$

Finally, evaluating the integrals $\chi, \alpha_{1}, \alpha_{2}$ and $\alpha_{3}$ permits us to obtain the translational velocity $\boldsymbol{U}$ of any ellipsoidal particle by combining (4.5) and (4.19).

\section{Application to the spheroidal particle}

This section examines the behaviour of a spheroidal particle whose surface is defined by the equation $x_{1}^{2}+x_{2}^{2}+x_{3}^{2} / \lambda^{2}=a^{2}$. We may consider a sphere of radius $a$ by setting $\lambda=1$ and either prolate $(\lambda>1)$ or oblate $(\lambda<1)$ spheroids. The case of prolate spheroids has been briefly addressed by using spheroidal coordinates and computing the electric field and the Stokes flow driven by the Lorentz body force in the whole fluid domain by C. Economou (Poster session, ICTAM Kyoto 1996).

\subsection{Predicted behaviour and discussion}

Before addressing the general spheroid, we look at the spherical case. In such circumstances, we obtain $\chi=2 a^{2}$ and $\alpha_{1}=\alpha_{2}=\alpha_{3}=2 / 3$. According to (4.17), the sum $T_{1}+T_{2}$ vanishes and the translational velocity $\boldsymbol{U}$ reads

$$
\boldsymbol{U}=-\frac{a^{2} C \sigma}{\mu}[\boldsymbol{E} \wedge \boldsymbol{B}], \quad C=\frac{1}{6} .
$$

The result (5.1) is as previously obtained by Leenov \& Kolin (1954) and Marty \& Alemany (1984).

A general spheroidal particle is either prolate $(\lambda>1)$ or oblate $(\lambda<1)$. Using Gradstheyn \& Ryzhik (1965, formulae 2.246, 2.266 and 2.269.2) and omitting the details, we arrive at the following non-zero Cartesian components of the 'mobility' pseudo-tensor $\boldsymbol{S}$

$$
\begin{aligned}
& S_{123}=-S_{213}=-a^{2} C_{1}(\lambda), \quad C_{1}(\lambda)=\frac{\alpha}{3[2-\alpha]}, \\
& S_{231}=-S_{132}=-a^{2} C_{2}(\lambda), \quad C_{2}(\lambda)=\frac{\alpha+\lambda^{2} \beta}{6[2-\beta]}, \\
& S_{312}=-S_{321}=-a^{2} C_{3}(\lambda), \quad C_{3}(\lambda)=\frac{\alpha+\lambda^{2} \beta}{6[2-\alpha]},
\end{aligned}
$$

with the definitions $\chi^{\prime}:=\chi / a^{2}, \alpha:=\alpha_{1}=\alpha_{2}, \beta:=\alpha_{3}$. By elementary algebra, we obtain

$$
\begin{gathered}
\alpha(\lambda)=\frac{\lambda^{2}}{\lambda^{2}-1}\left[1-\frac{\chi^{\prime}}{2 \lambda^{2}}\right], \quad \beta(\lambda)=\frac{1}{\lambda^{2}-1}\left[\chi^{\prime}-2\right], \\
\chi^{\prime}(\lambda)=\frac{\lambda}{\sqrt{\lambda^{2}-1}} \log \left[2 \lambda^{2}+2 \lambda \sqrt{\lambda^{2}-1}-1\right] \text { if } \lambda>1, \\
\chi^{\prime}(\lambda)=\frac{2 \lambda}{\sqrt{1-\lambda^{2}}} \arctan \left[\frac{\sqrt{1-\lambda^{2}}}{\lambda}\right] \text { if } \lambda<1 .
\end{gathered}
$$

As $\lambda \rightarrow 1$, we of course recover the previous case of a sphere of radius $a$ with $C_{k}(1)=C=1 / 6$ for $k \in\{1,2,3\}$. By virtue of (4.5) and previous results (5.2)-(5.4), the translational velocity $\boldsymbol{U}$ depends upon only three Cartesian components: $S_{123}, S_{231}$ 
and $S_{312}$. More precisely, we obtain

$$
\begin{gathered}
U_{1}=\frac{\sigma a^{2}}{\mu}\left\{C_{2}(\lambda) E_{3} B_{2}-C_{1}(\lambda) E_{2} B_{3}\right\}, \\
U_{2}=\frac{\sigma a^{2}}{\mu}\left\{C_{1}(\lambda) E_{1} B_{3}-C_{2}(\lambda) E_{3} B_{1}\right\}, \\
U_{3}=\frac{\sigma a^{2} C_{3}(\lambda)}{\mu}\left\{E_{2} B_{1}-E_{1} B_{2}\right\} .
\end{gathered}
$$

Accordingly, for any ambient fields $\boldsymbol{E}$ and $\boldsymbol{B}$,

$$
\boldsymbol{U} \cdot \boldsymbol{e}_{3}=\frac{\sigma}{\mu} S_{312}[\boldsymbol{E} \wedge \boldsymbol{B}] \cdot \boldsymbol{e}_{3}=-\frac{a^{2} C_{3}(\lambda) \sigma}{\mu}[\boldsymbol{E} \wedge \boldsymbol{B}] \cdot \boldsymbol{e}_{3} .
$$

As noted in Part 1, $\boldsymbol{U}$ is not necessarily parallel to $\boldsymbol{E} \wedge \boldsymbol{B}$. For example, if $\boldsymbol{E}=\boldsymbol{e}_{1}+\boldsymbol{e}_{3}$ and $\boldsymbol{B}=\boldsymbol{e}_{2}+\boldsymbol{e}_{3}$, we obtain

$$
\boldsymbol{U}=\frac{\sigma a^{2}}{\mu}\left\{C_{2}(\lambda) \boldsymbol{e}_{1}+C_{1}(\lambda) \boldsymbol{e}_{2}-C_{3}(\lambda) \boldsymbol{e}_{3}\right\}, \quad \boldsymbol{E} \wedge \boldsymbol{B}=\boldsymbol{e}_{3}-\boldsymbol{e}_{2}-\boldsymbol{e}_{1} .
$$

From Part 1, the vector $\boldsymbol{U}$ is known to be aligned with $\boldsymbol{E} \wedge \boldsymbol{B}$ only if $\boldsymbol{e}_{3}$ is parallel to $\boldsymbol{E} \wedge \boldsymbol{B}$ or lies in the plane normal to $\boldsymbol{E} \wedge \boldsymbol{B}$. Equalities (5.8)-(5.10) agree with these conditions and actually suggest splitting the latter circumstance into three different possibilities (Cases 1, 2 and 4 below) and to address the following cases

$$
\begin{aligned}
& \text { Case 1: } \boldsymbol{B} \| \boldsymbol{e}_{3} \text { or } \boldsymbol{E} \cdot \boldsymbol{e}_{3}=(\boldsymbol{E} \wedge \boldsymbol{B}) \cdot \boldsymbol{e}_{3}=0, \\
& \text { Case 2: } \boldsymbol{E} \| \boldsymbol{e}_{3} \text { or } \boldsymbol{B} \cdot \boldsymbol{e}_{3}=(\boldsymbol{E} \wedge \boldsymbol{B}) \cdot \boldsymbol{e}_{3}=0, \\
& \text { Case 3: } \boldsymbol{e}_{3} \| \boldsymbol{E} \wedge \boldsymbol{B}, \\
& \text { Case 4: }(\boldsymbol{E} \wedge \boldsymbol{B}) \cdot \boldsymbol{e}_{3}=0 \text { but not falling in Cases } 1 \text { or } 2 .
\end{aligned}
$$

These cases are illustrated in figure 2 and we indeed obtain the relations

$$
\boldsymbol{U}=-\frac{a^{2} C_{k}(\lambda) \sigma}{\mu}[\boldsymbol{E} \wedge \boldsymbol{B}] \text { for Case } k \in\{1,2,3\} .
$$

In Case 4, the velocity $\boldsymbol{U}$ admits a more tricky dependence (see (5.23)) upon $C_{1}(\lambda), C_{2}(\lambda)$ and angles $\theta=\left(\boldsymbol{E}, \boldsymbol{e}_{3}\right)$ and $\varphi=\left(\boldsymbol{B}, \boldsymbol{e}_{3}\right)$. Even when $\boldsymbol{U}$ is parallel to $\boldsymbol{E} \wedge \boldsymbol{B}$, the translational velocity depends upon the spheroid nature and the orientations of $\boldsymbol{E}$ and $\boldsymbol{B}$. We first emphasize this point by paying attention to Cases 1, 2 and 3. In Case 3, the velocity $\boldsymbol{U}$ depends solely upon the vector $\boldsymbol{E} \wedge \boldsymbol{B}$ as it is the case for a sphere and is parallel to $\boldsymbol{e}_{3}$. By contrast, if $(\boldsymbol{E} \wedge \boldsymbol{B}) \cdot \boldsymbol{e}_{3}=0$ with $\boldsymbol{E}$ or $\boldsymbol{B}$ normal to $\boldsymbol{e}_{3}$ (Case 1 or 2) the velocity $\boldsymbol{U}$ depends upon both $\boldsymbol{E}$ and $\boldsymbol{B}$. For instance, selecting $(\boldsymbol{E}, \boldsymbol{B})=\left(\boldsymbol{e}_{1}, \boldsymbol{e}_{3}\right)$ or $(\boldsymbol{E}, \boldsymbol{B})=\left(-\boldsymbol{e}_{3}, \boldsymbol{e}_{1}\right)$ we arrive at $\boldsymbol{U}=a^{2} C_{1}(\lambda) \sigma \boldsymbol{e}_{2} / \mu$ and $\boldsymbol{U}=a^{2} C_{2}(\lambda) \sigma \boldsymbol{e}_{2} / \mu$, respectively, for the same vector $\boldsymbol{E} \wedge \boldsymbol{B}=-\boldsymbol{e}_{2}$. As $\lambda \rightarrow \infty$, the prolate spheroid becomes a needle-shaped particle and we obtain

$$
C_{1}(\lambda)=\frac{1}{3}+O\left(\frac{\log \lambda}{\lambda^{2}}\right), C_{k}(\lambda)=\frac{2 \log \lambda-1}{6 \times 2^{3-k}}+O\left(\frac{\log ^{2} \lambda}{\lambda^{2}}\right) \text { for } k \in\{2,3\} .
$$

As $\lambda \rightarrow 0$, the oblate spheroid tends to a thin disk and this time the following asymptotic behaviours hold

$$
C_{1}(\lambda) \sim \frac{\pi}{12} \lambda, \quad C_{2}(\lambda) \sim \frac{1}{12}, \quad C_{3}(\lambda) \sim \frac{\pi}{24} \lambda .
$$


(a)

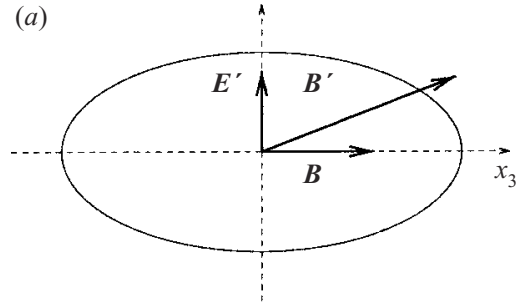

(c)

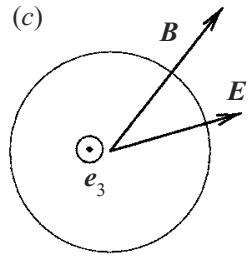

(b)
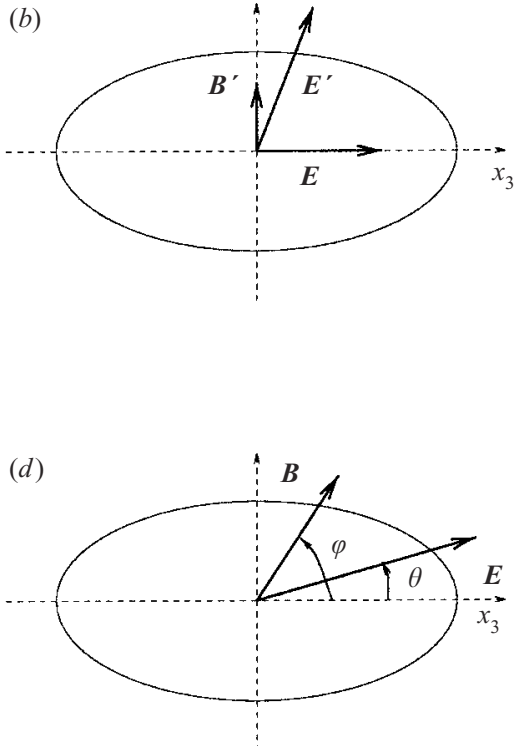

Figure 2. Cases 1, 2, 3 and 4 for a migration aligned with $\boldsymbol{E} \wedge \boldsymbol{B}$. (a) Possible settings $\boldsymbol{B} \| \boldsymbol{e}_{3}$ or $\left(\boldsymbol{E}^{\prime}, \boldsymbol{B}^{\prime}\right)$ in Case 1. (b) Possible settings $\boldsymbol{E} \| \boldsymbol{e}_{3}$ or $\left(\boldsymbol{E}^{\prime}, \boldsymbol{B}^{\prime}\right)$ in Case 2. (c) Case 3. (d) Case 4 with $\theta=\left(\boldsymbol{E}, \boldsymbol{e}_{3}\right)$ and $\varphi=\left(\boldsymbol{B}, \boldsymbol{e}_{3}\right)$.

Previous asymptotic approximations (5.18)-(5.19) clearly show that the behaviour of a given prolate or oblate spheroid strongly depends on the selected Case $k$ : for example, (5.19) reveals that a thin disk of radius $a$ does not move in Cases 1 and 3 whilst it translates at half the speed of a sphere of radius $a$ in Case 2. When looking at the sensitivity to the selected Case $k$, it is convenient to compare for each Case 1,2 or 3 the translational velocity $\boldsymbol{U}$ of our spheroid of volume $\mathscr{V}_{\mathscr{P}}=4 \pi \lambda a^{3} / 3$ to the velocity of the 'equivalent' sphere having the same volume (of radius $r=\lambda^{1 / 3} a$ ). Thus, we introduce the normalized functions

$$
c_{k}(\lambda):=a^{2} C_{k}(\lambda) /\left[r C_{k}(1)\right]=C_{k}(\lambda) /\left[C_{k}(1) \lambda^{2 / 3}\right], \quad k \in\{1,2,3\} .
$$

By virtue of (5.18) and (5.19), each function $c_{k}$ vanishes at infinity and at zero except the function $c_{2}$ which behaves like $\lambda^{-2 / 3} / 2$ as $\lambda \rightarrow 0$. The functions $c_{k}$, plotted in figure $3(a)$ for prolate spheroids and figure $3(b)$ for oblate spheroids, are found to be positive; any spheroid moves in the same direction as its 'equivalent' sphere, at least in any Case $k$. However, this motion depends upon both the spheroid nature (prolate or oblate) and the selected Case $k$. For example, as depicted in figure 3(a), any prolate spheroid translates slower than its 'equivalent' sphere in Cases 1 and 2. When both $\boldsymbol{E}$ and $\boldsymbol{B}$ are normal to $\boldsymbol{e}_{3}$ (Case 3 ) the prolate spheroid may either move faster (if $\lambda \leqslant \lambda_{3} \sim 12.549$ ) or slower (if it is slender enough with $\lambda \geqslant \lambda_{3}$ ) than its 'equivalent' sphere. For $\boldsymbol{E} \wedge \boldsymbol{B}$ of prescribed magnitude, any prolate spheroid moves faster in Case 3 than in other Cases 1 and 2. The smallest velocity is either obtained in Case 1 for a slender enough spheroid $\left(\lambda \geqslant \lambda_{c} \sim 3.117\right)$ or in Case 2 for other spheroids $\left(\lambda \leqslant \lambda_{c}\right)$. The difference $c_{1}(\lambda)-c_{2}(\lambda)$ actually only vanishes for a sphere $(\lambda=1)$ and a special prolate spheroid of critical slenderness ratio $\lambda=\lambda_{c}$.

As revealed by figure $3(b)$, the previous hierarchy is strongly modified for oblate spheroids. For any oblate spheroid, Cases 2 and 3 are found to induce the greatest and smallest translation, respectively, and the spheroid moves faster than its 'equivalent' 

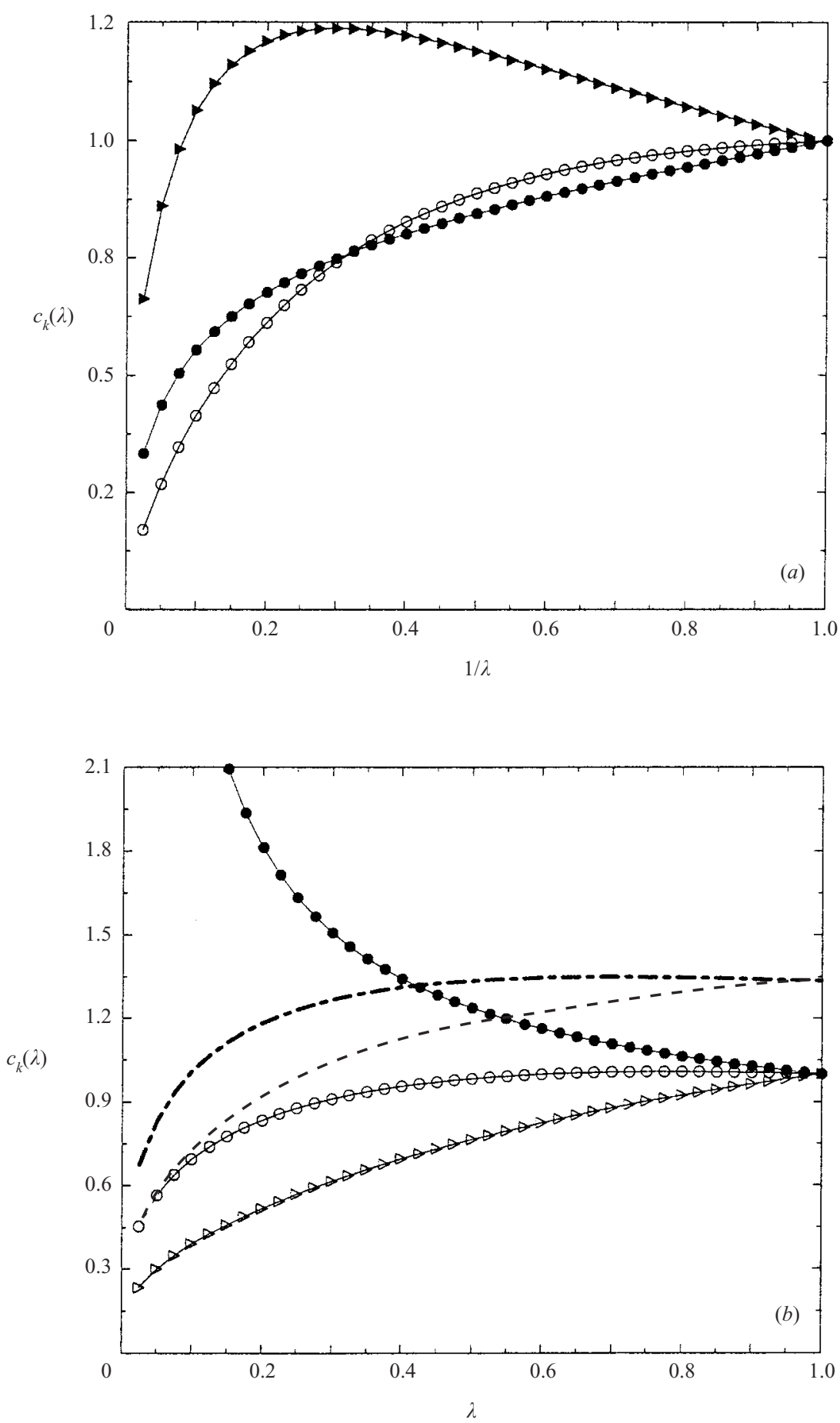

FIGURE 3. Normalized functions $c_{k}$ for Cases $k$. Case 1: $\bigcirc$; Case 2: $\bullet$ and Case 3: $\bullet(a)$ : Case of the prolate spheroid $\lambda \geqslant 1$. $(b)$ : Case of the oblate spheroid: $\lambda \leqslant 1$. Dot-dashed line: $c_{1 r}=c_{2 r}$ and dashed line: $c_{3 r}$. 
sphere in Case 2 only. In this latter case, the velocity becomes large, when compared to the case of the 'equivalent' sphere, as the prolate spheroid tends to a thin disk. This behaviour, not observed in other cases, deserves a more detailed analysis. As reflected by (4.5), each quantity $c_{k}(\lambda)$ sums up two contributions: a first term, denoted by $c_{k v}(\lambda)$, originates from the volume term arising in (4.5) and a second 'electric' term, denoted by $c_{k e}(\lambda)$, is associated with the perturbation potential (it relies on coefficients $\left.A_{i j k}^{\prime}\right)$. More precisely, we obtain:

$$
c_{1 v}(\lambda)=c_{2 v}(\lambda)=\frac{\chi^{\prime}+\alpha}{2 \lambda^{2 / 3}}, \quad c_{3 v}(\lambda)=\frac{\chi^{\prime}+\lambda^{2} \beta}{2 \lambda^{2 / 3}} .
$$

The above functions are plotted in figure $3(b)$ and comparisons with functions $c_{k}(\lambda)$ immediately gives the 'electric' term $c_{k e}(\lambda)=c_{k}(\lambda)-c_{k v}(\lambda)$. For instance, both functions $c_{1 e}$ and $c_{3 e}$ are negative in the whole range $\lambda \leqslant 1$. On the contrary, the 'electric' contribution $c_{2 e}$ is negative for $\lambda>\lambda_{2} \sim 0.4199$, zero for the critical value $\lambda=\lambda_{2}$ and positive for $\lambda<\lambda_{2}$. When $\lambda$ decreases, $c_{2 e}(\lambda)$ increases and tends to infinity because the non-zero electric field component $\boldsymbol{E} \cdot \boldsymbol{e}_{3}$ in Case 2 is nearly normal to the thin oblate spheroid and thereafter induces large derivatives of $\phi$ near the rim of the disk-shaped spheroid that result in large values of the 'electric' term.

In summary, even for the simple spheroidal shape, the translational velocity deeply depends upon the shape and the applied fields $\boldsymbol{E}$ and $\boldsymbol{B}$. In all Cases 1, 2 and 3, the velocity only vanishes with $\boldsymbol{E} \wedge \boldsymbol{B}$ and the spheroid moves in the same direction as its 'equivalent' sphere. Such conclusions might not hold for other circumstances. For instance, let us look at non-parallel ambient fields $\boldsymbol{E}=E_{i} \boldsymbol{e}_{i}$ and $\boldsymbol{B}=B_{i} \boldsymbol{e}_{i}$ that keep the spheroid motionless. According to (5.8)-(5.10), the translational velocity $\boldsymbol{U}$ may vanish only if $(\boldsymbol{E} \wedge \boldsymbol{B}) \cdot \boldsymbol{e}_{3}=0$ and $\boldsymbol{E} \cdot \boldsymbol{e}_{3} \neq 0$ (see above Case 1). The combination of (5.8)-(5.9) shows that if $E_{1}=E_{2}=0$ then $U_{1}=U_{2}=0$ but $\boldsymbol{E}_{\wedge} \boldsymbol{B}=\mathbf{0}$. Thus, for symmetry reasons we assume that $E_{1}=0, E_{2}>0, E_{3}>0$ and denote by $0<\theta<\pi / 2$ the angle between vectors $\boldsymbol{e}_{3}$ and $\boldsymbol{E}_{\infty}$. Under such assumptions, $U_{3}$ vanishes only if $B_{1}=0$ (because quantities $c_{k}(\lambda)$ are non-zero) and we introduce the angle $\varphi$ between vectors $\boldsymbol{e}_{3}$ and $\boldsymbol{B}_{\infty}$ with $0<\varphi \leqslant \pi / 2$. These notations are illustrated in figure $2(d)$. We thus have $U_{3}=U_{2}=0$ and require $U_{1}=0$. This latter condition reads $\varphi=\varphi_{c}$ where the critical angle $\varphi_{c}$ is given, for any setting $(\lambda, \theta)$, by the equation

$$
\tan \varphi_{c}=\frac{C_{1}(\lambda)}{C_{2}(\lambda)} \tan \theta=\frac{c_{1}(\lambda)}{c_{2}(\lambda)} \tan \theta
$$

For any pair $(\lambda, \theta)$ the equation (5.22) indeed admits a unique solution $\varphi_{c}$. Note that $\varphi_{c}=\theta$ if $c_{1}(\lambda)=c_{2}(\lambda)$, i. e. for the sphere $(\lambda=1)$ or the previously encountered critical prolate spheroid $\left(\lambda=\lambda_{c}\right)$. We discard these two special cases for which the electric and magnetic fields are parallel (the product $\boldsymbol{E} \wedge \boldsymbol{B}$ vanishes and the 'equivalent' sphere is motionless) and compare for $\varphi \neq \theta$ the velocities $\boldsymbol{U}$ and $\boldsymbol{U}_{s}$ of the spheroid and its 'equivalent' sphere. Actually, for $\varphi \neq \theta$, we obtain

$$
\boldsymbol{U}=6 C_{1}(\lambda) f(\varphi) \boldsymbol{U}_{s}, \quad f(\varphi)=1+\left(1-\frac{C_{2}}{C_{1}}\right) \frac{\cos \theta \sin \varphi}{\sin (\theta-\varphi)}, \quad \theta=\left(\boldsymbol{E}_{\infty}, \boldsymbol{e}_{3}\right), \quad \varphi=\left(\boldsymbol{B}_{\infty}, \boldsymbol{e}_{3}\right) .
$$

Accordingly, in Case 4 (see definition (5.16)) any non-spherical spheroid of slenderness ratio $\lambda \neq \lambda_{c}$ also translates parallel to the 'equivalent' sphere (as in Cases 1, 2 and $3)$, i. e. as soon as the electric field is not normal to a plane of symmetry and all vectors $\boldsymbol{e}_{3}, \boldsymbol{E}$ and $\boldsymbol{B}$ lie in the same plane (which is a plane of symmetry). In addition, 
a straightforward calculation yields

$$
\frac{\mathrm{d} f}{\mathrm{~d} \varphi}=\left[1-\frac{C_{2}}{C_{1}}\right] \frac{\sin \theta \cos \theta}{\sin ^{2}(\theta-\varphi)} .
$$

Thus, the function $f$ changes sign at the critical value $\varphi=\varphi_{c}$ and the spheroid and its 'equivalent' sphere may either translate parallel or anti-parallel (at least in vicinity of $\left.\varphi_{c}\right)$.

\subsection{Taking into account gravity}

So far, gravity has been neglected in our analysis. However, it is straightforward to include gravity effects, at least for any orthotropic particle of uniform density $\rho_{s}$ embedded in a liquid metal of uniform density $\rho$. For any orthotropic particle and uniform densities $\rho_{s}$ and $\rho$, the action of gravity field $\boldsymbol{g}$ indeed results in a zero torque relative to the particle centre of volume $O$ of the particle and a net force

$$
\boldsymbol{F}^{(4)}=\mathscr{V}_{\mathscr{P}}\left(\rho_{s}-\rho\right) \boldsymbol{g} .
$$

The force $\boldsymbol{F}^{(4)}$ now enters in equation (2.17) and again $\boldsymbol{\Omega}=\mathbf{0}$. Thus, the freely suspended orthotropic particle translates at velocity $\boldsymbol{U}^{\prime}$ such that

$$
\boldsymbol{U}^{\prime}=\boldsymbol{U}+\boldsymbol{U}^{s}, \quad \boldsymbol{U}^{s} \cdot \boldsymbol{e}_{i}=\frac{\left(\rho_{s}-\rho\right) \mathscr{V}_{\mathscr{P}}}{\mu K_{i i}} \boldsymbol{g} \cdot \boldsymbol{e}_{i} .
$$

In (5.26), there is no summation over suffixes $i$, and $\boldsymbol{U}$ and $\boldsymbol{U}^{s}$ designate the velocity without gravity and the usual sedimentation velocity, respectively. For a given orthotropic particle, it thereafter seems possible to select the applied fields $\boldsymbol{g}, \boldsymbol{E}$ and $\boldsymbol{B}$ so that the particle does not move. For example, for a sphere of radius $a$, the sedimentation velocity $\boldsymbol{U}^{s}$ admits the simple and well-known form

$$
\boldsymbol{U}^{s}=\frac{2 a^{2}}{9 \mu}\left(\rho_{s}-\rho\right) \boldsymbol{g},
$$

and, by virtue of (5.1), the sphere does not translate whenever the following link holds

$$
\left(\rho_{s}-\rho\right) \boldsymbol{g}=\frac{3}{4} \sigma[\boldsymbol{E} \wedge \boldsymbol{B}] .
$$

For other orthotropic particles, the answer is not trivial and actually depends on the relative orientation of the particle and the external fields $\boldsymbol{E}$ and $\boldsymbol{B}$. In order to illustrate this feature, we again consider the case of a spheroid, of shape parameter $\lambda$, and restrict ourselves to the previously defined Cases 1,2 or 3. Under these assumptions, two circumstances arise:

(i) For Cases 1 and 2, the vectors $\boldsymbol{E} \wedge \boldsymbol{B}$ and therefore the required gravity field $\boldsymbol{g}$ are normal to $\boldsymbol{e}_{3}$. Hence, the sedimentation velocity of the spheroid and the required condition read

$$
\boldsymbol{U}^{s}=\frac{a^{2}\left(\rho_{s}-\rho\right)}{12 \mu}\left[\chi^{\prime}+\alpha\right] \boldsymbol{g}, \quad\left(\rho_{s}-\rho\right) \boldsymbol{g}=h_{k}(\lambda) \sigma[\boldsymbol{E} \wedge \boldsymbol{B}], \quad h_{k}(\lambda)=\frac{12 C_{k}(\lambda)}{\chi^{\prime}+\alpha} .
$$

(ii) For Case 3, both vectors $\boldsymbol{E} \wedge \boldsymbol{B}$ and $\boldsymbol{g}$ are parallel to $\boldsymbol{e}_{3}$. This time, the sedimentation velocity and the required relation become

$$
\boldsymbol{U}^{s}=\frac{a^{2}\left(\rho_{s}-\rho\right)}{12 \mu}\left[\chi^{\prime}+\lambda^{2} \beta\right] \boldsymbol{g}, \quad\left(\rho_{s}-\rho\right) \boldsymbol{g}=h_{3}(\lambda) \sigma[\boldsymbol{E} \wedge \boldsymbol{B}], \quad h_{3}(\lambda)=\frac{12 C_{3}(\lambda)}{\chi^{\prime}+\lambda^{2} \beta} .
$$



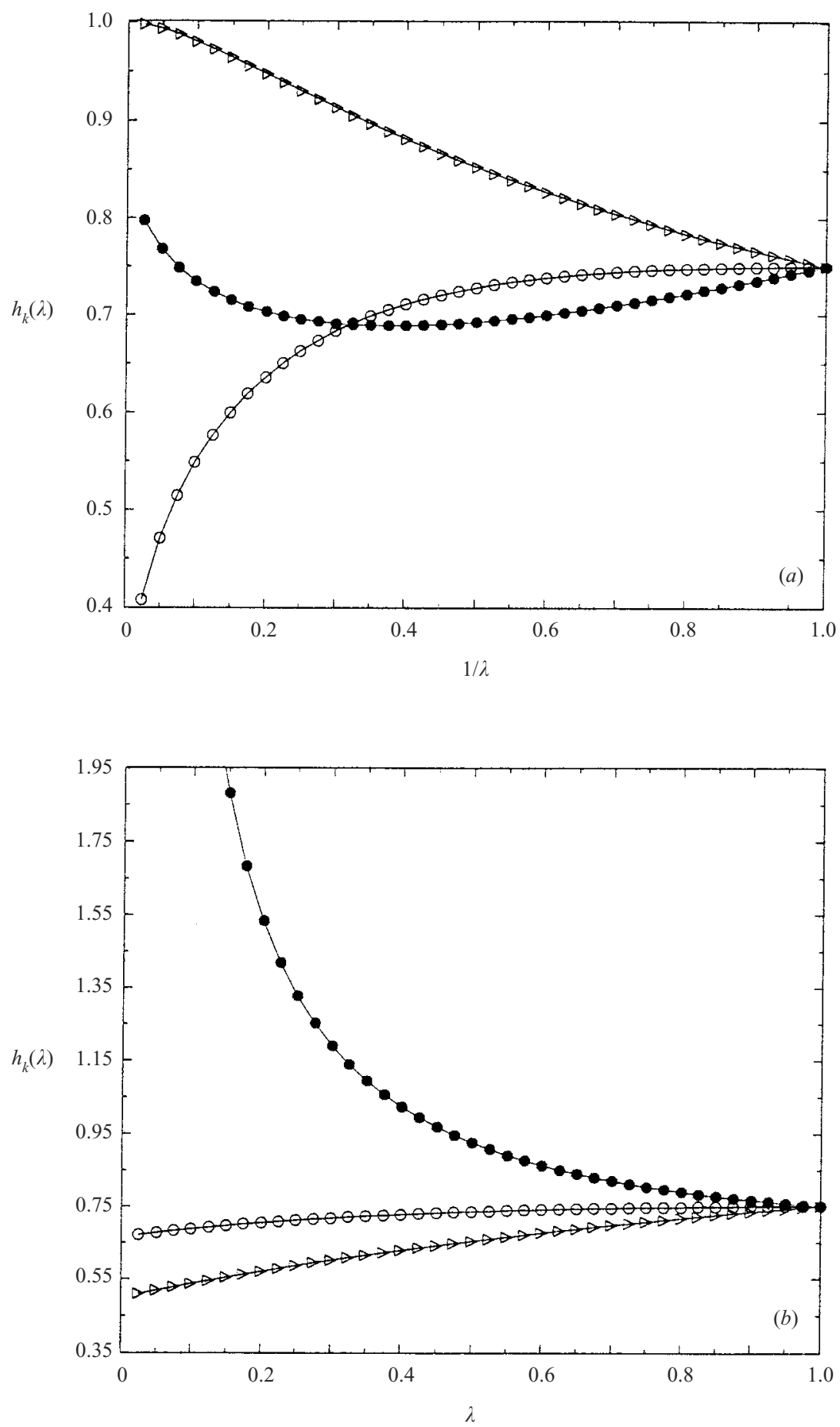

Figure 4. Normalized functions $h_{k}$ for Cases $k$. Case 1: $\bigcirc$; Case 2: $\bullet$ and Case 3:

(a) Case of the prolate spheroid: $\lambda \geqslant 1$. (b) Case of the oblate spheroid: $\lambda \leqslant 1$. 
The non-dimensional function $k_{k}$ actually compares the magnitude of the translational velocity $\boldsymbol{U}$ and settling velocity $\boldsymbol{U}^{s}$ for identical body forces $\left(\rho_{s}-\rho\right) \boldsymbol{g}$ and $\sigma[\boldsymbol{E} \wedge \boldsymbol{B}]$ in Case $k, k \neq 4$. In view of (5.18) and (5.19), these functions admit the following asymptotic behaviours:

$$
\begin{aligned}
& h_{1}(\lambda) \sim \frac{2}{3}, h_{2}(\lambda) \sim \frac{2}{3 \pi \lambda}, h_{3}(\lambda) \sim \frac{1}{2} \text { as } \lambda \rightarrow 0, \\
& h_{1}(\lambda) \sim \frac{2}{\log \lambda}, h_{2}(\lambda) \sim 1, h_{3}(\lambda) \sim 1 \text { as } \lambda \rightarrow \infty .
\end{aligned}
$$

Of course (recall (5.28)), $h_{k}(1)=3 / 4$. The functions $h_{k}$ are plotted in figure $4(a)$ for prolate spheroids and in figure $4(b)$ for oblate spheroids. Under equal body forces $\left(\rho_{s}-\rho\right) \boldsymbol{g}$ and $\sigma[\boldsymbol{E} \wedge \boldsymbol{B}]$, the prolate spheroid is seen to settle faster for all Cases 1,2 and 3 as illustrated by figure $4(a)$. Case 3 always induces the greatest translational velocity $\boldsymbol{U}$ and the ordering of Cases 1 and 2 depends on the selected slenderness ratio $\lambda$ with the same critical value $\lambda_{c}$ as for figure $3(a)$ (indeed $h_{1}-h_{2}$ vanishes with $c_{1}-c_{2}$ ). As depicted in figure 4(b), any oblate spheroid settles faster in Cases 1 and 3 whereas it may both settle faster for $\lambda>\lambda_{4} \sim 0.4199$ or slower for $\lambda<\lambda_{4}$ in Case 2 . As the oblate spheroid approaches the thin disk of radius $a$ it moves much faster than under the gravity field. This behaviour is again related to the 'electric' term arising in function $c_{2}$ that becomes large in such instance. Finally, any given oblate spheroid experiences its faster and weaker velocities in Case 2 and 3, respectively.

\section{Conclusions}

A rigid particle of arbitrary shape may both rotate and translate under the application of ambient electric field $\boldsymbol{E}$ and magnetic field $\boldsymbol{B}$. The boundary formulation advocated in $\S 3$ applies to the case of any particle and seems well adapted for a numerical approximation of the required rigid-body motion.

As proved in Part 1, any isotropic particles translates (with no rotation) parallel to $\boldsymbol{E} \wedge \boldsymbol{B}$. Non-isotropic but orthotropic particles also only transate but exhibit a more subtle behaviour: the translational velocity $\boldsymbol{U}$ is found to depend greatly upon both the shape and the ambient fields $(\boldsymbol{E}$ and $\boldsymbol{B})$ orientations and is not systematically aligned with $\boldsymbol{E} \wedge \boldsymbol{B}$. This dramatic sensitivity has been analysed and discussed in detail for both oblate and prolate spheroidal particles. Depending upon $(\boldsymbol{E}, \boldsymbol{B})$, the behaviours of a spheroid and its 'equivalent' sphere (having the same volume) may strongly differ and the spheroid may even be motionless with $\boldsymbol{E} \wedge \boldsymbol{B} \neq \mathbf{0}$.

In future, it would be nice to deal with non-orthotropic particles, for instance pear-shaped particles. Such particles both translate and rotate. This challenging task makes use of numerics and is under current investigation.

The author is indebted to Professor H. K. Moffatt for his valuable suggestions and comments on this work.

\section{Appendix A. Derivation of (3.4)-(3.8)}

By virtue of (3.1) we first note that

$$
-8 \pi \mu u_{L}^{(i)}(M)=\left\{\int_{S}\left[\boldsymbol{f}_{L}^{(i)} \cdot \boldsymbol{e}_{k}\right](P) G_{j k}(P, M) \mathrm{d} S_{P}\right\} \boldsymbol{e}_{j} .
$$


Accordingly, the definition (3.3) of $I_{L}^{(i)}(r)$ becomes

$$
I_{L}^{(i)}(r)=\int_{\Omega(r)}\left[\int_{S}\left[\boldsymbol{f}_{L}^{(i)} \cdot \boldsymbol{e}_{k}\right](P) G_{j k}(P, M) \mathrm{d} S_{P}\right] \boldsymbol{e}_{j} \cdot(\nabla \phi \wedge \boldsymbol{B})(M) \mathrm{d} \Omega,
$$

and switching the integrations over $\Omega(r)$ and $S$, we obtain

$$
I_{L}^{(i)}(r)=\int_{S}\left[\boldsymbol{f}_{L}^{(i)} \cdot \boldsymbol{e}_{k}\right](P) J_{k}^{P}(r) \mathrm{d} S_{P}, \quad J_{k}^{P}(r)=\int_{\Omega(r)} G_{j k}(P, M) a_{j}(M) \mathrm{d} \Omega,
$$

with $a_{j}(M):=\boldsymbol{e}_{j} \cdot[\nabla \phi \wedge \boldsymbol{B}](M)$. Adopting the notation $g_{, l}=\partial g / \partial x_{l}$ and $g_{, j l}=$ $\partial^{2} g / \partial x_{j} \partial x_{l}$, it is straightforward to check that, for $P \in S$ and $M \in \Omega(r)$,

$$
G_{j k}(P, M)=F_{j, l l}^{k}(P, M)-F_{l, l j}^{k}(P, M), \quad F_{j}^{k}(P, M)=\delta_{j k} P M .
$$

Recall that $\boldsymbol{B}$ is uniform and $\phi$ is harmonic in $\Omega$. Thus, $a_{j, j}=0$ in $\Omega$. Exploiting (A 4$)$ for $M$ in $\Omega(r)$, it follows that

$$
G_{j k}(P, M) a_{j}(M)=P M_{, l l} a_{k}(M)-\left[P M_{, k} a_{j}\right]_{, j}(M) .
$$

Since $a_{k}(M)=\epsilon_{k m n}\left[\boldsymbol{B} \cdot \boldsymbol{e}_{n}\right] \phi_{, m}(M)$, it easily follows that

$$
P M_{, l l} a_{k}(M)=\epsilon_{k m n}\left[\boldsymbol{B} \cdot \boldsymbol{e}_{n}\right]\left\{\left(\phi_{, m}(M) P M_{, l}-\phi_{, m l}(M) P M\right)_{, l}+P M \phi_{, m l l}(M)\right\} .
$$

Since $\boldsymbol{B}$ is uniform and $\phi_{, l l}=0$ in $\Omega$, (A 5) and (A 6) yield $G_{j k}(P, M) a_{j}(M)=$ $[\nabla \cdot \boldsymbol{A}](M)$ in $\Omega(r)$ with

$$
A_{l}(M)=A_{l}=\boldsymbol{A}(M) \cdot \boldsymbol{e}_{l} \epsilon_{k m n}\left[\boldsymbol{B} \cdot \boldsymbol{e}_{n}\right]\left\{\phi_{, m}(M) P M_{, l}-\phi_{, m l}(M) P M\right\}-P M_{, k} a_{l}(M) .
$$

Observe that $A_{l}$ decays at least as fast as $1 / r^{3}$ far from the particle. Using the divergence theorem to convert the volume integral $J_{k}^{P}(r)$ into a surface integral over $S \cup S_{r}$ and letting the radius $r$ tend to infinity, we finally arrive at the key decomposition (3.4).

\section{Appendix B. Derivation of equalities (4.13) and (4.17)}

Following Lamb (1932), we introduce for any point $M\left(x_{1}, x_{2}, x_{3}\right)$ the three roots $\nu_{e}<\mu_{e}<\lambda_{e}$ of equation (4.6), considered as a cubic in $\lambda$. Inside and outside the ellipsoid we respectively obtain $\lambda_{e}<0$ and $\lambda_{e}>0$ while $\lambda_{e}=0$ on the surface $\mathscr{E}_{0}$. For any constant values $a$ and $b$, the function $\psi_{a, b}(M)=\psi_{a, b}\left(\lambda_{e}\right)$, defined by

$$
\begin{aligned}
& \psi_{a, b}(M)=a \int_{0}^{\infty} \frac{\mathrm{d} t}{\Delta(t)}+b x_{1} \int_{0}^{\infty} \frac{\mathrm{d} t}{\left(a_{1}^{2}+t\right) \Delta(t)} \text { if } \lambda \leqslant 0, \\
& \psi_{a, b}(M)=a \int_{\lambda_{e}}^{\infty} \frac{\mathrm{d} t}{\Delta(t)}+b x_{1} \int_{\lambda_{e}}^{\infty} \frac{\mathrm{d} t}{\left(a_{1}^{2}+t\right) \Delta(t)} \text { if } \lambda_{e} \geqslant 0,
\end{aligned}
$$

is harmonic in $\mathbb{R}^{3} \backslash \mathscr{E}_{0}\left(\right.$ Lamb 1932) and continuous across $\mathscr{E}_{0}$. Accordingly, it admits in the whole space the basic single-layer representation

$$
\psi_{a, b}(M)=\int_{\mathscr{E}_{0}} \frac{d(P)}{P M} \mathrm{~d} S_{P}, \quad d(P)=\frac{s(P)}{2 \pi a_{1} a_{2} a_{3}}\left[a+b \frac{x_{1}}{a_{1}^{2}}\right],
$$

where the surface density $d$ has been calculated by using, on $\mathscr{E}_{0}$, the basic relations (no summation over suffixes $l$ )

$$
\left[\left(\frac{\partial \lambda_{e}}{\partial x_{l}}\right)_{x_{j \neq l}}\right]_{\lambda_{e}=0}=\frac{2 x_{l} s^{2}(M)}{a_{l}^{2}}, \quad n_{l}(M)=\left[\boldsymbol{n} \cdot \boldsymbol{e}_{l}\right](M)=s(M) \frac{x_{l}}{a_{l}^{2}} .
$$


Combining (4.10),(4.11) and (4.14) we thus obtain, for any point $M$ belonging to the ellipsoid $\mathscr{E}_{0}$,

$$
\psi_{1,0}=\int_{\mathscr{E}_{0}} \frac{s(P) \mathrm{d} S_{P}}{2 \pi a_{1} a_{2} a_{3} P M}=\frac{\chi}{a_{1} a_{2} a_{3}}, \quad \psi_{0,1}=\int_{\mathscr{E}_{0}} \frac{x_{1}^{P} s(P) \mathrm{d} S_{P}}{2 \pi a_{1}^{3} a_{2} a_{3} P M}=\frac{\alpha_{1} x_{1}}{a_{1} a_{2} a_{3}} .
$$

Since $\psi_{1,0}$ and $\psi_{0,1}$ are harmonic inside the particle and constant or linear on $\mathscr{E}_{0}$, it follows that (B 5) holds within the whole domain $\mathscr{P} \cup \mathscr{E}_{0}$. Resorting to cyclic permutation of suffixes, we thereafter obtain (4.13), both inside the particle and on its boundary.

Now we look at the sum $T_{1}+T_{3}$. For $\lambda>0$, let us denote by $V_{\lambda}$ the volume that is confined by the ellipsoids $\mathscr{E}_{0}$ and $\mathscr{E}_{\lambda}$. Invoking the divergence theorem and definition (4.14) of function $\phi^{\prime}$, we obtain

$$
T_{1}+T_{3}=\frac{a_{1} a_{2} a_{3}}{\left[\alpha_{2}-2\right]} B_{1} E_{2} A^{(3)}\{P(\lambda)-N(\lambda)\}
$$

with surface integral $P(\lambda)$ and volume integral $N(\lambda)$ defined as follows

$$
P(\lambda)=\int_{\mathscr{E}_{\lambda}}\left[\phi_{, 2}^{\prime} I_{l}-\phi^{\prime}, 2 l J\right]\left[\boldsymbol{n} \cdot \boldsymbol{e}_{l}\right] \mathrm{d} S, \quad N(\lambda)=\int_{V_{\lambda}}\left\{\phi_{, 2}^{\prime} I_{l}-\phi_{, 2 l}^{\prime} J\right\}, l d \Omega .
$$

The charge density $d^{\prime}$ associated to the potential $\phi^{\prime}$ (see (B 3)) obeys

$$
d^{\prime}(P)=s(P) x_{2} /\left[2 \pi a_{1} a_{2}^{3} a_{3}\right], \quad \int_{\mathscr{E}_{0}} d^{\prime}(P) \mathrm{d} S_{P}=0, \quad \int_{\mathscr{E}_{0}} x_{i}^{P} d^{\prime}(P) \mathrm{d} S_{P}=2 \delta_{i 2} / 3 .
$$

Accordingly, the function $\phi_{, 2}^{\prime}$ admits the following asymptotic behaviour

$$
\phi_{, 2}^{\prime}=2\left(x_{1}^{2}+x_{3}^{2}-2 x_{2}^{2}\right) /\left[3 r^{5}\right]+O\left(1 / r^{4}\right) \text { as } r=O M \rightarrow \infty .
$$

In addition, as $\lambda$ tends to infinity, the ellipsoid $\mathscr{E}_{\lambda}$ becomes a sphere of radius $\lambda^{1 / 2}$ and $\boldsymbol{n} \cdot \boldsymbol{e}_{l} \sim x_{l} / r$ whilst (look at definitions (4.11)) functions $I_{l}$ and $J$, respectively, behave like $x_{l} / r$ and $r$. Accordingly, $P(\lambda)$ vanishes as $\lambda$ goes to infinity. Noting that $\phi_{, 2}^{\prime}$ is harmonic and $J,_{l}=I_{l}$ in the whole domain $V_{\lambda}$, the required volume integral $N(\lambda)$ becomes

$$
N(\lambda)=\int_{V_{\lambda}} \phi_{, 2}^{\prime} I_{l, l} \mathrm{~d} \Omega=2 \int_{V_{\lambda}}\left[\int_{\mathscr{E}_{0}} \frac{s(P) \mathrm{d} S_{P}}{P M}\right] \phi_{, 2}^{\prime} \mathrm{d} \Omega=D \int_{V_{\lambda}}\left[\int_{\lambda_{e}}^{\infty} \frac{\mathrm{d} t}{\Delta(t)}\right] \phi_{, 2}^{\prime} \mathrm{d} \Omega,
$$

with $M\left(x_{1}, x_{2}, x_{3}\right)$ identified by its orthogonal curvilinear coordinates $\nu_{e}<\mu_{e}<\lambda_{e}$ and $D:=4 \pi a_{1} a_{2} a_{3}$. The last relations in (B 10) have been obtained by differentiating, in $V_{\lambda}$, our definition (4.11) and finally applying (B 2)-(B 3). In addition to our orthogonal curvilinear coordinates $v_{e}<\mu_{e}<\lambda_{e}$, we introduce three related and positive functions $h_{1}, h_{2}$ and $h_{3}$ as detailed in Lamb (1932, $\left.\S 112\right)$. Note that $\Xi\left(\mu_{e}\right)<0$ whereas $\Xi\left(v_{e}\right)>0$ and $\Xi\left(\lambda_{e}\right)>0$ almost everywhere outside the ellipsoidal particle. Within the domain $V_{\lambda}$, the volume element reads $\mathrm{d} \Omega=\mathrm{d} \nu_{e} \mathrm{~d} \mu_{e} \mathrm{~d} \lambda_{e} /\left[h_{1} h_{2} h_{3}\right]$ and the reader may also check that $2\left(\partial \lambda_{e} / \partial x_{2}\right)_{x_{1}, x_{3}}=x_{2} h_{1}^{2} /\left[a_{2}^{2}+\lambda_{e}\right]$ with

$$
\frac{2 x_{2}^{2} h_{1}}{h_{2} h_{3}\left[a_{2}^{2}+\lambda_{e}\right] \Delta\left(\lambda_{e}\right)}=\frac{\left(\mu_{e}-v_{e}\right)\left(a_{2}^{2}+\mu_{e}\right)\left(a_{2}^{2}+v_{e}\right)}{\Delta\left(v_{e}\right) \sqrt{-\Xi\left(\mu_{e}\right)}\left(a_{2}^{2}-a_{1}^{2}\right)\left(a_{2}^{2}-a_{3}^{2}\right)}=F\left(\mu_{e}, v_{e}\right) .
$$


By virtue of (4.14) and (B 11), the relation (B 10) becomes

$$
\frac{N(\lambda)}{4 \pi a_{1} a_{2} a_{3}}=\int_{0}^{\lambda}\left[W_{0} W_{1} K\right]\left(\lambda_{e}\right) \mathrm{d} \lambda_{e}-\left\{\int_{0}^{\lambda} \frac{W_{0}\left(\lambda_{e}\right)}{a_{2}^{2}+\lambda_{e}}\left[\int_{\mu_{e}} \int_{v_{e}} \frac{F\left(\mu_{e}, v_{e}\right)}{4} \mathrm{~d} \mu_{e} \mathrm{~d} v_{e}\right] \mathrm{d} \lambda_{e}\right\}
$$

with, for $m \in\{0,1\}$, the definitions

$$
W_{m}\left(\lambda_{e}\right):=\int_{\lambda_{e}}^{\infty} \frac{\mathrm{d} t}{\left(a_{2}^{2}+t\right)^{m} \Delta(t)}, K\left(\lambda_{e}\right):=\int_{\mu_{e}} \int_{v_{e}} \frac{\mathrm{d} \mu_{e} \mathrm{~d} v_{e}}{h_{1} h_{2} h_{3}} .
$$

On the ellipsoid $\mathscr{E}_{\lambda}$ the surface element $\mathrm{d} S$ reads $\mathrm{d} S=\mathrm{d} \mu_{e} \mathrm{~d} v_{e} /\left[h_{2} h_{3}\right]$ and $h_{1}=$ $2 s(M)$ (see Lamb 1932) on $\mathscr{E}_{0}$. Integrating the relation (B 11) on the surface $\mathscr{E}_{0}$, we immediately obtain

$$
\int_{\mu_{e}} \int_{v_{e}} \frac{F\left(\mu_{e}, v_{e}\right)}{4} \mathrm{~d} \mu_{e} \mathrm{~d} v_{e}=\int_{\mathscr{E}_{0}} \frac{2 x_{2}^{2} h_{1}}{\Delta(0) a_{2}^{2}} \mathrm{~d} S=\frac{4 \pi}{3} .
$$

Observing that the volume of the domain enclosed by the ellipsoid $\mathscr{E}_{\lambda}$ is equal to $4 \pi \Delta(\lambda) / 3$, we arrive at

$$
\int_{V_{\lambda}} \mathrm{d} \Omega=\int_{0}^{\lambda} K\left(\lambda_{e}\right) \mathrm{d} \lambda_{e}=4 \pi[\Delta(\lambda)-\Delta(0)] / 3, \quad 3 K\left(\lambda_{e}\right)=4 \pi \frac{\mathrm{d} \Delta}{\mathrm{d} \lambda_{e}} .
$$

Integrating by parts the first integral on the right-hand side of (B 12) and taking into account definitions (B 13) makes it possible to obtain

$$
\frac{N(\lambda)}{4 \pi a_{1} a_{2} a_{3}}=\frac{4 \pi}{3}\left\{\left[W_{0} W_{1} \Delta\right]\left(\lambda_{e}\right)-\left[W_{0} W_{1} \Delta\right](0)+\int_{0}^{\lambda} W_{1}\left(\lambda_{e}\right) \mathrm{d} \lambda_{e}\right\} .
$$

Integrating by parts once more and finally letting $\lambda$ tend to infinity, we arrive at equality (4.17).

\section{REFERENCES}

Beskos, D. E. 1987 Introduction to Boundary Element Methods. In Computational Methods in Mechanics (ed. D. E. Beskos). Elsevier.

Bonnet, M. 1999 Boundary Integral Equation Methods for Solids and Fluids. John Wiley.

Brebbia, C. A., Telles, J. C. L. \& Wrobel, L. C. 1984 Boundary Element Techniques. Springer.

Gradshteyn, I. S. \& Ryzhik, I. M. 1965 Tables of Integrals, Series and Products. Academic.

Happel, J. \& Brenner, H. 1973 Low Reynolds Number Hydrodynamics. Martinus Nijhoff.

JEFFERY, G. B. 1922 The motion of ellipsoidal particles immersed in a viscous fluid. Proc. R. Soc. Lond. A 102, 161-179.

Kim, S. \& Karrila, S. J. 1991 Microhydrodynamics: Principles and Selected Applications. Butterworth.

Ladyzhenskaya, O. A. 1969 The Mathematical Theory of Vicous Incompressible Flow. Gordon \& Breach.

Lamb, H. 1932 Hydrodynamics, 6th edn. Cambridge University Press.

Leenov, D. \& Kolin, A. 1954 Theory of electromagnetophoresis. I. Magnetohydrodynamic forces experienced by spherical and symmetrically oriented cylindrical particles. J. Chem. Phys. 22, 683-688.

Marty, P. \& Alemany, A. 1984 Theoretical and experimental aspects of electromagnetic separation. In Metallurgical Applications of Magnetohydrodynamics (ed. H. K. Moffatt \& M. R. E. Proctor), pp. 245-259. Metals Society.

Moffatt, H. K. \& Sellier, A. 2002 Migration of an insulating particle under the action of uniform ambient electric and magnetic fields. Part 1. General theory. J. Fluid Mech. 464, 279-286.

Pozrikidis, C. 1992 Boundary Integral and Singularity Methods for Linearized Viscous Flow. Cambridge University Press.

Teubner, M. 1982 The motion of charged particles in electric fields. J. Chem. Phys. 76, 5564-5573. 